\title{
Diagnostics utiles et inutiles ou: parfois less is more
}

\author{
Hans Stalder
}

Prof. Dr méd., membre de la rédaction

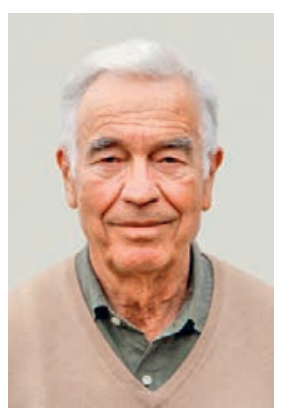

Pour résoudre un problème médical, il faut faire une bonne anamnèse, un bon status, poser un diagnostic différentiel et si nécessaire demander des examens complémentaires pour confirmer le diagnostic final. Un bon médecin sait faire de bons diagnostics! Autrefois on s'exerçait avec les case reports du New England et on éprouvait une grande satisfaction d'arriver au diagnostic avant d'avoir tourné la page donnant le diagnostic pathologique.

Bien sûr, établir un diagnostic fait toujours partie de l'activité médicale, mais une fois qu'il est posé, c'est le suivi qui prédomine, surtout en ce qui prévaut aujourd'hui: les maladies chroniques.

Soyons modestes: souvent nous utilisons des mots tirés du grec ou du latin pour qualifier les plaintes du patient: les maux de gorge deviennent une pharyngite, les douleurs du dos une lombalgie, l'élévation du cholestérol une hypercholestérolémie, etc. Ça donne un air savant, mais ne demande pas un effort intellectuel particulièrement poussé.

Faire un diagnostic exact est par ailleurs souvent inutile. Cela nous semble évident en cas de rhume: une fois exclu le streptocoque pourquoi préciser l'étiologie virale si de toute façon ça n'a pas d'importance? C'est moins évident dans des situations plus compliquées. Faut-il vraiment avoir recours à une imagerie sophistiquée en cas de démence chez une personne âgée, une fois exclues les rares causes traitables (les red flags), comme un manque de vitamine $\mathrm{B}_{12}$, une hypothyroïdie ou une dépression, si l'on ne dispose de toute façon pas d'un traitement?

Souvent nous ne posons donc pas la question essentielle: le résultat de l'examen complémentaire - positif ou négatif - va-t-il changer ma prise en charge? Deux histoires rapportées récemment par des connaissances peuvent servir d'exemples. Un médecin demande pour un confrère de 80 ans, diabétique, traité oralement, un CAC scoring, la détection du stock de calcium dans les artères coronaires par CT. En cas de résultat pathologique il aurait demandé une coronarographie. Il a négligé de discuter les suites possibles de l'examen avec le malade, qui de toute façon aurait refusé une telle in- tervention. L'autre personne a consulté le spécialiste pour une hypoacousie unilatérale après une grippe. Un neurinome ayant été exclu par IRM, le spécialiste propose un enregistrement des potentiels évoqués. Lorsque la patiente lui demande si le résultat aura une incidence sur le traitement, sa réponse est négative. Des examens complémentaires sont aussi superflus si le diagnostic clinique est soit d'emblée évident ou extrêmement peu probable: inutile de faire un ECG chez une jeune fille ayant une douleur thoracique localisée, car la probabilité que le résultat soit un faux positif est plus grande que celle qu'il soit un vrai positif, ce qui entraînera des investigations ultérieures potentiellement dangereuses.

Demander un examen complémentaire peut être nocif. Quand nous demandons une radio pour une lombalgie aiguë après avoir exclu les red flags, le diagnostic radiologique ne correspondra souvent pas au tableau clinique. Transmettre cette trouvaille au malade risque de chroniciser ses douleurs [1].

Personnellement je trouve les recommandations du mouvement "choosing wisely" un peu simplistes: 5 do not's par société médicale... Ne faudrait-il pas plutôt nous inciter à réfléchir: mon attitude dépendra-t-elle du résultat de l'examen complémentaire (positif vs négatif)? Avant de le prescrire ai-je discuté les différentes suites possibles avec le patient? Est-ce que je demande cet examen seulement pour satisfaire ma curiosité (et je ne parle même pas du portemonnaie)? Est-ce que le résultat peut avoir un effet nocif? Partager de telles réflexions avec le patient peut être ardu, surtout avec celui qui grâce à l'internet croit tout savoir mieux que nous, mais discuter et convaincre est après tout une des parties nobles de notre travail.

«Un bon médecin sait ce qu'il faut faire, un excellent ce qu'il ne faut pas faire.» Ce vieux dicton s'applique aussi au diagnostic et il est aujourd'hui d'autant plus approprié que les examens complémentaires sont devenus bien plus sophistiqués (et chers).

\section{Référence}

1 Srinivas SV, Deyo RA, Berger ZD. Application of "Less Is More" to low back pain. Arch Intern Med. 2012;172:1016-20. 